The Nepali Mathematical Sciences Report, Vol. 37, No.1 \& 2, 2020: 1-13

DOI: https://doi.org/10.3126/nmsr.v37i1-2.34063

\title{
ON THE TRANSIT-BASED EVACUATION STRATEGIES IN AN INTEGRATED NETWORK TOPOLOGY
}

\author{
ISWAR MANI ADHIKARI $^{1}$ AND TANKA NATH DHAMALA ${ }^{2}$ \\ 1 Tribhuvan University, Prithvi Narayan Campus, Pokhara, Nepal \\ 2 Tribhuvan University, Central Department of Mathematics, Kathmandu, Nepal
}

\begin{abstract}
Evacuation planning problem deals with sending the maximum number of evacuees from the danger zone to the safe zone in minimum time as efficiently as possible. The dynamic network flow models for various evacuation network topology have been found suitable for the solution of such a problem. Busbased evacuation planning problem (BEPP), as an important variant of the vehicle routing problem (VRP), is one of the emerging evacuation planning problems. In this work, an organized overview of this problem with a focus on their solution status is compactly presented. Arrival patterns of the evacuees including their transshipments at different pickup locations and their assignments are presented. Finally, a BEPP model and a solution for a special network are also proposed.
\end{abstract}

Key Words: Evacuation planning problem; evacuation network topology; bus-based evacuation

AMS (MOS) Subject Classification. 90B10, 90B20.

\section{INTRODUCTION}

Evacuation planning problem deals with sending the maximum number of evacuees from source (danger zone) to sink (safe zone) in minimum time as efficiently as possible. It can be further classified into microscopic and macroscopic planning. The microscopic planning deals with the individual evacuee's behavior in which some probabilistic laws for individual evacuees movement are presented and mainly based on the simulation approaches. But in macroscopic planning, they are principally based on optimization approaches where the evacuees are treated as the homogeneous group and only the common characteristics are considered. Optimization approaches on such macroscopic evacuation planning can further be classified like a heuristic approach, population optimization, modeling as fluid dynamics, traffic management, optimal evacuation destination, and network flow formulation. Among them, the dynamic network flow formulation has been found suitable evacuation optimization approach. There has been a fair amount of work regarding different aspects of network flow formulation related to the evacuation planning problem, as referred by [2], [3], [6]-[8]. For detail about the time minimization on such problems with minimum clearance time in an integrated evacuation network topology, we refer to [1], 4] and the references therein.

Received: August 4, 2020 Accepted/Published Online: January, 2021. 
For an evacuation network, a remarkable BEPP model was formulated by Bish in [5] to minimize the time of evacuation using a given number of homogeneous buses satisfying all evacuee demands, without violating both sink and vehicle capacity constraints. Here, the number of evacuees at the demand node might be greater than the capacity of a bus and requests the split delivery within the pickup locations. But for a BEPP in [12], the number of evacuees at every source node be known in terms of the integral multiples of the busloads and does not request for the split delivery service. However, the split delivery need not improve always the evacuation duration, as mentioned in [4].

A robust bus-based evacuation planning problem RBEPP has been presented in [11] in which only some possible scenarios of the evacuees are provided rather than the exact number in each of the collection points. Based on such BEPP and RBEPP, Pyakurel et al. 223] explored a wide horizon to the research related to the transit-dependent evacuation planning problem. Kathmandu, one of the densely populated city, has been considered as the disaster region. In their solutions, they have used the branch and bound approach presented in [12, and the tabu search heuristic from [11], respectively. In their results, the domain of optimal solutions remains on a large number of buses with higher capacity and speed, irrespective of the population chosen where the choice of the number of sources and sinks does not play a significant role. A risk-based bus schedule technique for pickup location with concerns of disaster dynamics and time-varying supply-demand conditions is proposed in [17].

This paper considers optimization problems in evacuation planning. However, covering the broad horizon of the evacuation strategies respecting to such problems in a single paper is almost impossible. Here, we are focused fundamentally on the time minimization aspect of the transshipment problems in the transit-based evacuation scenarios in the integrated evacuation network topology. A compact and systematic overview of the evacuation strategies based on network flow formulation with regard to their solution status is presented. Besides this, it has a glimpse of the arrival patterns of the evacuees in such the evacuation network and their assignment with reference to a bus-based evacuation planning problem.

The paper is organized as follows. Section 2 presents an organized overview of evacuation network topology, different variants the network flow models and their solution strategies. Collection of evacuees at different pickup locations in an evacuation scenario is presented Section 3. Bus based evacuation planning problem is in Section 4. Finally, Section 5 concludes the paper.

\section{Evacuation Network Topology}

Consider a dynamic network $\mathcal{N}=(G, u, \tau, S, Z)$ that consists of a directed graph $G=(V, A)$ in which an arc $e=(i, j) \in A$ has a flow capacity function $u: A \rightarrow \mathbb{R}_{+}$ and a transit time function $\tau: A \rightarrow \mathbb{R}_{+}$. Here, $S$ and $Z$ are used to denote the set of sources (danger zones) and sinks (shelters or safe zones), respectively. Their union, i.e. $S \cup Z$ denotes the terminals. It may additionally provided with supply/demand function $\nu: S \cup Z \rightarrow \mathbb{R}_{+}$with supplies $\nu(s)>0$ for all sources $s \in S$ and demands $\nu(z)<0$ for all 
sinks $z \in Z$ such that $\sum_{s \in S \cup Z} \nu(s)=0$. One of the fundamental problems in the dynamic network is the evacuation planning problem.

In a dynamic network flow model, the time can be considered continuous or discrete. In the case of a static network flow model, the time parameter is absent. In general, the continuous model yields more accurate results over the discrete but are more challenging to compute. Evacuees movement can be considered as pedestrian or car-based (auto-based) or bus-based (transit-based). In most of the large cities, many people depend on transitvehicles, say buses, and are not provided with their own vehicles. The great loss of people in disasters is due to a lack of proper planning of transit-vehicles rather than the disaster itself. In such situations, transit-based evacuation system is more effective than others, in general, and can be operated in an integrated approach in a different network topology. However, it may depend upon the nature of the evacuation scenario. The nature of the pickup locations may also differ from each other. Such pickup locations can also be prioritized depending upon their locations and availabilities.

2.1. Prioritized network. A prioritized network is a multi-terminal network which consists prioritized terminals. Under the given priority of terminals, two flows can be compared according to departure/arrival flows form/in the sinks or sources. A flow value is said to be lexicographic if it is compared according to the rank of the terminals. Let $\mathcal{N}=(G, u, \tau, S, Z)$ be a prioritized network with priority $t_{1}, t_{2}, \ldots, t_{n} ; t_{i} \in S \cup Z$. Let

$$
|f|_{t}:= \begin{cases}\sum_{e \in A_{t}^{+}} f_{e}, & t \in S \text { is a source } \\ \sum_{e \in A_{t}^{-}} f_{e}, & t \in Z \text { is a sink }\end{cases}
$$

be the out/in flow value from/in the source and sink, respectively. Suppose $f^{1}$ and $f^{2}$ be the terminal respecting flows, $f^{1}$ is said to be lexicographically bigger than $f^{2}$ and written as $f^{1} \geq_{L} f^{2}$ if $\exists l \in\{0,1, \ldots, k-1\}: \forall i \in\{1,2, \ldots, l\}:\left|f^{1}\right|_{t_{i}}=\left|f^{2}\right|_{t_{i}} \wedge\left|f^{1}\right|_{t_{l+1}}>\left|f^{2}\right|_{t_{l+1}}$ or $\forall i \in\{1,2, \ldots, k\}:\left|f^{1}\right|_{t_{i}}=\left|f^{2}\right|_{t_{i}}$. The maximum flow respecting the rank of the terminals is said to be lexicographically maximum flow.

A solution to maximum flow problem sends the maximal amount of flow from the sources to the sinks for the fixed integer time horizon $T$. Lexicographically maximum (lexmax) flow problem with many sources and many sinks was introduced and many efficient algorithms were presented from different aspects in [19, 20, 21]. In such a problem, the terminals (sources and/or sinks) are ordered with certain priority for a lex-max flow respecting the priority, considering the set of sources and sinks be prioritized as $s_{1}, s_{2}, \ldots, s_{k}$ and $y_{1}, y_{2}, \ldots, y_{k}$, respectively, as a prioritized network. Such a lex-max flow is not necessarily a maximum flow and vice versa, however, they are equivalent for two-terminal networks [10].

In the quickest transshipment problem, a given number of evacuees has to be shifted in minimum time. Such problem have been studied by the help of the lex-max dynamic flow problem applying the minimum cost flow computations as a tool, in such a prioritized network. For a given time $\mathrm{T}$ and an ordered set of multi-terminals, the lex-max dynamic flow problem finds a feasible flow that lexicographically maximizes the flow leaving each terminal 
in the prioritized network. Hoppe and Tardos [13] deals with a chain decomposable flows in such a network.

A chain flow $\gamma=\langle\nu, \pi\rangle$ is a static flow of values $\nu \geq 0$ along the path $\pi$ in a network $\mathcal{N}=(G, u, \tau, S, Z)$. The length of chain flow $\tau(\gamma)$ also represents total length of path $\tau(\pi)$. Given the time horizon T no less than $\tau(\gamma)$, any chain flow $\gamma$ induces a dynamic flow by sending $\nu$ units of flow along the path $\pi$ at every time step until $T-\tau(\gamma)$. A proper chain flow starts and ends at terminals. A multiset of proper chain flows, $\Gamma=\left\{\gamma_{1}, \gamma_{1}, \ldots, \gamma_{k}\right\}$ is a chain decomposition of static flows $f$ if $\sum_{i=1}^{k} \gamma_{i}=f$. It becomes a standard chain decomposition of $f$ if all chain flows in it use edges in the same direction as of $f$. A flow decomposition with zero flows on all cycles is the path decomposition. One may assume that there is no flow along any cycle as the opposite flows along all cycles could be canceled.

In the non-standard chain decomposition, the chain flows may use oppositely directed flows on edges. It may use a residual edge with negative transit times. For $e=(i, j)$, a unit of flow sent from $i$ at time $\theta$ reaches $j$ at time $\theta+\tau_{e}$ is nothing other than sending a negative unit of flow from $j$ at time $\theta+\tau_{e}$ to reach $i$ at time $\theta$. Let $\gamma_{1}^{\prime}$ be the another chain that flows through $(j, i)$, then that cancels the chain flow $\gamma_{1}$ along $(i, j)$. The chain decomposable flows do not violate capacity constraints. Moreover, the non-standard chain decomposition induces the dynamic flows.

Starting with zero flow, the lex-max flow algorithm computes the successive layers of minimum cost static flows in the residual network of the previous layers and adds standard chains to the existing one. It takes $k$ times the complexity of minimum cost flow computations, for a given time $T$.

Theorem 2.1. [13] A k-terminal lex-max dynamic flow problem can be solved in polynomialtime complexity with $O(k . g(m n))$, where $O(g(m n))$ is required for one minimum cost flow computation on a network with $n$ nodes and $m$ edges.

2.2. Arc reversal network. The arc reversal is an approach that modifies the orientation of arcs in the network to increase outbound flow with reduced time on the evacuation. It is an effective and widely accepted approach for the optimal use of available road network in evacuation management that increases the outward road capacities from the disastrous areas towards the safe destination.

Let the reversal of an arc $e=(i, j)$ be $e^{\prime}=(j, i)$, then the transformed network of $\mathcal{N}$ consists of the modified arc capacities and constant transit times as,

$$
u_{\bar{e}}=u_{e}+u_{e^{\prime}} \text { and } \tau_{\bar{e}}= \begin{cases}\tau_{e}, & \text { if } e \in A \\ \tau_{e^{\prime}}, & \text { otherwise }\end{cases}
$$

where an edge $\bar{e} \in \bar{A}$ in a transformed network, if $e \vee e^{\prime} \in A$ in $\mathcal{N}$. The remaining graph structure and data are unaltered. For the solution status of a problem, we have

Theorem 2.2. [28] The maximum static $s-z$ flow problem with arc reversals is polynomially solvable. 
Proof. The maximum static $s-z$ flow problem on arc reversal in the transformed network can be solved by decomposing the obtained flow into paths and cycles, and by deleting the latter one assuming that the arcs on either direction will never be used in the optimal flow. An $\operatorname{arc} e^{\prime}=(j, i) \in A$ is reversed if and only if the flow on $(i, j)$ is greater than $u_{e}(i, j)$, or if there is a nonnegative flow along $(i, j) \notin A$ and the resulting flow is maximum with arc reversal. Such a flow is feasible and optimal too with polynomial-time complexity.

And a similar result follows in a multi-terminal network by a simple reduction.

Theorem 2.3. [28] The maximum static flow problem on the arc reversal network with multiple sources and sinks is polynomially solvable.

Proof. As the general maximum static flow problem on arc reversal can be reduced to the respective $s^{*}-z^{*}$ flow problem, providing the super-source $s^{*}$ and super-sink $z^{*}$. Here, $s^{*}$ is connected to each $s \in S$ having arc capacities equal to their respective surplus and $z^{*}$ to each $z \in Z$ having arc capacities equal their respective deficits. Hence, the respective static version of the maximum flow problem with arc reversal with multiple sources and multiple sinks is also polynomially solvable.

The similar situation exists regarding the maximum dynamic flow problem on arc reversal in two-terminal networks, though in general, we have,

Theorem 2.4. 28] The maximum dynamic flow problem in a multi-terminal network on arc reversal is $\mathcal{N} \mathcal{P}$-complete.

Such $\mathcal{N} \mathcal{P}$-completeness in a multi-terminal network is so due to the conflict with reverting the intermediate arcs, [28]. Hence, numerous heuristics and metaheuristics have been presented and implemented for the solutions of different types of evacuation planning problems by arc reversal approach, [26]. Instead of full arc reversal, arcs are better to reverse up to the necessary capacity only, as the partial arc reversal. Such an approach has presented lucidly with different models, algorithms, and solution strategies by Pyakurel et al. [25, 24]. In recent work, authors in [4, have integrated it with the earliest arrival transshipment and the flows in zero transit times as,

Theorem 2.5. [4] The earliest arrival evacuee problem having zero transit times with partial arc reversal capability follows the principle of temporally repeated flows and can be solved in polynomial-time complexity.

Proof. The flow that reached to each of the pickup locations at zero transit times determines the maximum number of evacuees at every possible time instance from the beginning, as in [29]. So, it follows the principle of temporally repeated flows on the transformed network which is equivalent to the solution with arc reversals capability on the original network, as in [27]. Hence, it can be solved in polynomial-time complexity.

For the limited resources it is not possible to select all arc reversals as demanded by the optimal arc reversal strategy, as each arc reversal is associated with certain operating costs. To address such issues, its budget constraint version is investigated in [9]. They have solved such a problem in a time-expanded network so it is pseudo-polynomial. 
Theorem 2.6. 9] The maximum dynamic flow problem with budget constraint switching cost can be solved optimally in pseudo-polynomial-time.

2.3. Integrated evacuation network. Depending upon nature and needs, different networks can be embedded to have an integrated network. For example, the authors in [14] had presented an integrated contraflow network for multimodal evacuation. They integrate the non-contraflow, the full-lane-contraflow, and the bus-contraflow networks to shorten the strategy set up time, to maximize the evacuation network capacity, and to realize the transit cycle operation, respectively. Adhikari et al. 4] have presented an integrated evacuation strategy in an embedded network topology, $\mathcal{N}=\mathcal{N}_{1} \cup \mathcal{N}_{2}$, where $\mathcal{N}_{1}$ consists of directed two-way network respecting the partial arc reversal capability for the collection of evacuees. By treating such supplies as the sources, the available set of transit-vehicles at the depot are assigned to transverse the evacuees in the dominant routing to the sinks in $\mathcal{N}_{2}$. In such an approach, evacuees are collected at the pickup locations from the sources in the earliest arrival flow pattern at zero transit times and then they are assigned to the transit-vehicles in the embedded network with minimum clearance time.

Theorem 2.7. [4] The transit-vehicle assignment algorithm for an integrated evacuation network gives the dominating solution for the transit-vehicle assignment problem with minimum clearance time.

\section{Collection of evacuees at pickup locations}

The collection of evacuees at pickup locations can be categorized to follow different arrival patterns. But two of the prominent BEPP formulations as in [12] and [5] have assumed the evacuees to be at the pickup locations with no specific arrival patterns. Such a problem is extended to an RBEPP by assuming that the number of evacuees is not known exactly but a set of estimates for the number of evacuees at each source is given [11]. Evacuees have gathered themselves at different pickup locations relative to the population density of the transit-dependent people nearby them in [23] with no specific arrival patterns. A constant arrival rate of evacuees has been considered by the authors in [22. Such an assumption is still unrealistic as the true arrival process is probabilistic. A linear programming mathematical model using binary variables was developed in [16] to select the most suitable location and the number of bus stops.

The cumulative percentage of total evacuees loaded in the evacuation network by time $\theta$ since the start of the evacuation can be estimated as [15,

$$
\xi(\theta)=\frac{1}{1+\exp [-\alpha(\theta-h)]} .
$$

Here, $\alpha$ is the loading rate of evacuees representing the response of the public to the disaster. The parameter $h$ is the half loading time, represented by the mid-position of such curve. Let $\xi^{i}\left(\theta_{k}\right)$ and $\xi^{i}\left(\theta_{k-1}\right)$ be the cumulative percentage of the evacuees arrival at the pickup location $i$ at the end of time interval $k$ and $k-1$, respectively. Let $\delta_{i}$ be the number of evacuees arriving at pickup location $i$ during the evacuation time frame and $\theta_{k}$ is the 
end of the evacuation time interval at $k$ and $Q$ be the maximal passenger capacity of the vehicle. Let $d_{i}^{k}$ be the number of demand of vehicles at eack pickup locations $i$ during the time interval $k$, then the number of evacuees arriving at pickup location $i$ during the evacuation time frame is,

$$
\delta_{i}=\frac{d_{i}^{k} \times Q}{\xi^{i}\left(\theta_{k}\right)-\xi^{i}\left(\theta_{k-1}\right)} .
$$

The flow of evacuees from the disaster zone $s \in S$ to the pickup locations $p \in P$ over time is a non-negative function $f$ on $A \times \mathbb{R}_{+}$, for given time $\mathbf{T}=\{0,1, \ldots, T\}$ satisfying the flow conservation and capacity constraints 3.3 .3 .5 . The inequality flow conservation constraints allow waiting for flow at intermediate nodes. However, the flow conservation constraints force that flows entering an intermediate node must leave it again immediately.

$$
\begin{aligned}
\sum_{\sigma=\tau_{e}}^{T} \sum_{e \in A_{i}^{\text {in }}} f\left(e, \sigma-\tau_{e}\right)-\sum_{\sigma=0}^{T} \sum_{e \in A_{i}^{\text {out }}} f(e, \sigma)=0, \forall i \notin\{S, P\}, \\
\sum_{\sigma=\tau_{e}}^{\theta} \sum_{e \in A_{i}^{\text {in }}} f\left(e, \sigma-\tau_{e}\right)-\sum_{\sigma=0}^{\theta} \sum_{e \in A_{i}^{\text {out }}} f(e, \sigma) \geq 0, \forall i \notin\{S, P\}, \theta \in \mathbf{T}, \\
0 \leq f(e, \theta) \leq u_{e}, \quad \forall e \in A, \theta \in \mathbf{T} .
\end{aligned}
$$

Here, $A_{i}^{\text {out }}=\{e=(i, j) \in A\}$ and $A_{i}^{i n}=\left\{e^{\prime}=(j, i) \in A\right\}$ are the sets of outgoing and incoming arcs, respectively for the node $i \in V$. For $s$, the flow value be $\nu_{f}(s)>0$, and for $p$ it becomes $\nu_{f}(p)<0$, whereas $\sum_{i \in V} \nu_{f}(i)=0$. If the supply and demand on such terminals be fixed for all $i \in\{s, p\}$, then the earliest arrival evacuee problem maximizes value $\left(\nu_{f}, \theta\right)$ for all $\theta \in \mathbf{T}$, as in Equation (3.6) satisfying the constraints (3.3) 3.5 ).

$$
\left(\nu_{f}, \theta\right)=\sum_{\sigma=0}^{\theta} \sum_{e \in A_{s}^{\text {out }}} f(e, \sigma)=\sum_{\sigma=\tau_{e}}^{\theta} \sum_{e \in A_{p}^{\text {in }}} f\left(e, \sigma-\tau_{e}\right) .
$$

Let $\nu(P, \theta)$ stands for the flow amount out of source $s$ that reaches to the pickup location $\mathrm{P}$ at time $\theta \in Z_{+}$with zero transit times, then the total flow amount out of $s$ that reached to $P$ for all time up to $\theta^{\prime} \in \mathbb{Z}_{+}$, with $\tau_{e}=0$, is given by

$$
\left|\nu_{f}\right|_{\theta^{\prime}}=\sum_{\theta=1}^{\theta^{\prime}}|\nu(P, \theta)| .
$$

For the given time bound $T$, the value in Equation 3.7 becomes

$$
\left|\nu_{f}\right|=\sum_{\theta=1}^{T}|\nu(P, \theta)|
$$


3.1. Transshipment problems. When preparing for an evacuation, it is uncertain how much time that will take to enact it. So, it is preferential to plan for the transversal of a maximum number of evacuees reaching safety not only at the ultimate clearance time but also in each possible time unit, i.e. with the maximum possible value of the value $\left(\nu_{f}, \theta\right)$ for all $\theta \in \mathbf{T}$, as in Equation (3.6). It is offered by the earliest arrival flows. On the other hand, the quickest transshipment problem is to find the minimum clearance time to send a given amount of flow from multiple sources to multiple sinks. Each of the earliest arrival transshipment additionally optimizes the amount of flow leaving the network at all times and is therefore the quickest transshipment. But not necessarily the converse, as illustrated in Example 3.1. Moreover, the earliest arrival transshipment does not necessarily exist in the networks with multiple sinks, but the quickest transshipments do.

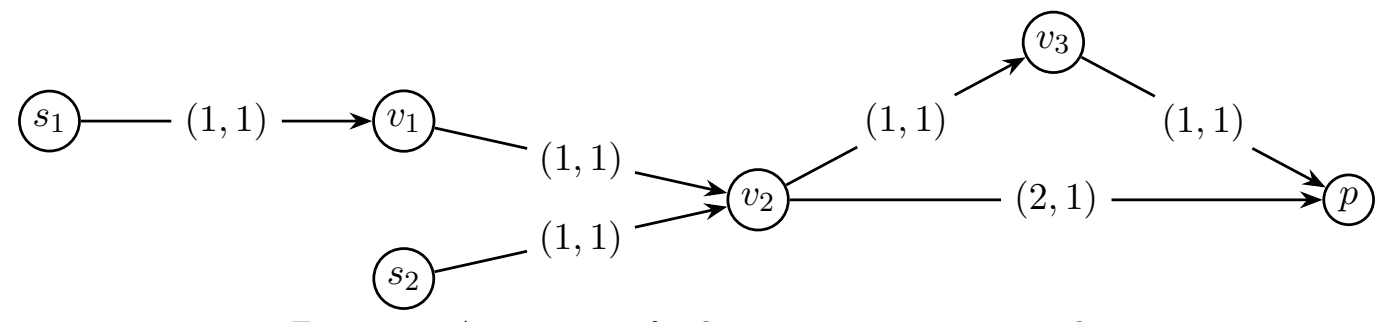

Figure 1. An instance of a dynamic evacuation network.

Example 3.1. Consider a dynamic network $\mathcal{N}$ having $\left(u_{e}, \tau_{e}\right)$ be the capacity and transit time for $e \in A$ as in Figure 1. For $s_{1}$ and $s_{2}$ be the sources, and $p$ be the pickup location, let $\nu\left(s_{1}\right)=\nu\left(s_{2}\right)=3$ and $\nu(p)=-6$.

Consider two different flow patterns with their respective time, path assignments, flow value, and the total flow in different columns, as in Table 1 and Table 2. Here, Table 1 represents a quickest transshipment that is not an earliest arrival transshipment. But, Table 2 represents an earliest arrival transshipment which is also a quickest transshipment.

TABle 1. A quickest transshipment which is not an earliest arrival transshipment.

\begin{tabular}{|c|c|c|c|}
\hline Time unit & Path assignment & flow value & Total flow reached \\
\hline \multirow{2}{*}{3} & $s_{1}-v_{1}-v_{2}-p$ & 1 & \multirow{2}{*}{2} \\
\cline { 2 - 4 } & $s_{2}-v_{2}-v_{3}-p$ & 1 & 2 \\
\hline \multirow{2}{*}{4} & $s_{1}-v_{1}-v_{2}-p$ & 1 & \multirow{2}{*}{4} \\
\cline { 2 - 4 } & $s_{2}-v_{2}-v_{3}-p$ & 1 & 4 \\
\hline \multirow{2}{*}{5} & $s_{1}-v_{1}-v_{2}-p$ & 1 & \multicolumn{2}{|c}{6} \\
\cline { 2 - 4 } & $s_{2}-v_{2}-v_{3}-p$ & 1 & 6 \\
\hline
\end{tabular}

\section{Bus BASED EVACUATION PlanNing PROBLEM}

In large cities of the developing countries, many people depend on transit-vehicles, say buses. They are to be given a special attention due to their ages, language ineffciencies, different health problems, or other physical disabilities. The great loss of people in disasters is due to a lack of proper planning of transit-vehicles rather than the disaster itself. It was 
TABLE 2. An earliest arrival transshipment which is also a quickest transshipment.

\begin{tabular}{|c|c|c|c|}
\hline Time unit & Path assignment & flow value & Total flow reached \\
\hline 2 & $s_{2}-v_{2}-p$ & 1 & 1 \\
\hline \multirow{2}{*}{3} & $s_{2}-v_{2}-p$ & 1 & \multirow{2}{*}{3} \\
\cline { 2 - 3 } & $s_{1}-v_{1}-v_{2}-p$ & 1 & 3 \\
\hline \multirow{2}{*}{4} & $s_{2}-v_{2}-p$ & 1 & \multirow{2}{*}{5} \\
\cline { 2 - 3 } & $s_{1}-v_{1}-v_{2}-p$ & 1 & 5 \\
\hline 5 & $s_{1}-v_{1}-v_{2}-p$ & 1 & 6 \\
\hline
\end{tabular}

noticed that, the great loss of people on Hurricane Katrina was due to the lack of proper planning for the transit-based evacuees an mentioned in [18. In an integrated evacuation network, the formulation of a prominent BEPP and its dominant assignment plays a vital role. Based on the BEPP formulation by [5], one of the prominent version of BEPP has been considered by [12] as follows:

Let $\left(\tau_{i j}\right)_{i \in P, j \in Z}$ be a matrix of source-sink travel times. Let the vectors $\tau_{d i},\left(l_{i}\right)_{i \in P}$, and $\left(\mu_{j}\right)_{j \in Z}$ be the depot-source travel times, number of evacuees, and sink capacities, respectively. Then the BEPP is to find a tour plan to minimize the maximum travel times overall buses such that all the evacuees be transported to the sinks.

For this, it is assumed that the number of evacuees at every source node be known in terms of the integral multiples of the busloads and is so for the sink. For the solution of such BEPP, the branch and bound algorithms with four upper bounds and three lower bounds for time, three branching rules to minimize the number of branches and two tree reduction strategies to avoid the equivalent branches have been presented by Goerigk et al. in [12]. Upper bounds have been constructed in polynomial-time complexity by four heuristic algorithms. Among the lower bounds, the first one is based on the estimation of the travel times from sources to sinks and from sinks to sources, respectively. The second lower bound is based on the fact that lower bound for the maximum travel time is the average travel time. The third one is about the simplification of model formulation.

For $i \in P$ and $j \in Z$, consider $\tau_{i j}, \tau_{d i}, l_{i}$ and $\mu_{j}$ be as in such BEPP as formulated in [12]. For $k \in \mathbb{R}$, is there a tour plan with $\mathcal{T}_{\max } \leq k$ ? Regarding its complexity, we have,

Theorem 4.1. [11] The decision version of BEPP is $\mathcal{N P}$-complete, even if $\tau_{d i}=0$ and $\tau_{i j}=\tau_{i^{\prime} j}$ for all $i, i^{\prime} \in P$ and $j \in Z$.

Proof. The BEPP is reduced to the scheduling problem of scheduling $n$-jobs on $P$-parallel machines, which is $\mathcal{N} \mathcal{P}$-hard, as mentioned in [11]. Such a scheduling problem with maximum completion time, $C_{\max } \leq k$ for a given $k$ has a yes-instance if and only if the respective bus routing plan of BEPP with $\mathcal{T}_{\max } \leq k$ has a yes-instance. As both the completion time and the feasibility of the given solution can be checked polynomially, the decision version of BEPP is $\mathcal{N} \mathcal{P}$-complete.

4.1. BEPP in a diminished evacuation network. Consider a BEPP for a diminished evacuation network close to the real scenario which is equivalent to the third lower 
bound, [12] as about the simplification of model formulation. Here, sinks are far away from the dangerous zone. The bus depot is in a closed environment to the disaster zone with $\mathrm{B}$ be the set of available buses. So, let the super pickup node with the bus depot be $Y_{0}$ with the evacuees $\xi_{y_{0}}$. Assume the capacity of each bus be 1 . The movement between $Z$ is ignored. The set of tours of the buses cannot be changed anymore after they start to move and are connected. The maximum number of trips for the evacuation process is given by $\xi_{y_{0}}$. The nonnegative travel cost of $\tau_{0 j}$ on each edge $e=(0, j) \in E$ are taken symmetric. Let the sinks $z \in Z$ be at a distance of $\tau_{0 j}$ from $Y_{0}$, where $y_{j}^{b}$ denotes a tour that the bus b drives from the source to sink $j \in Z$ and back from the sink $j \in Z$ to the source. Let $\mathcal{T}_{\max }$ be the duration of evacuation overall buses, then the problem becomes,

$$
\begin{aligned}
\underset{\text { minimize }}{\mathcal{T}_{\max }} & \\
\text { such that } \mathcal{T}_{\max } & \geq \sum_{j \in Z} \tau_{0 j} y_{j}^{b} \forall b \in B, \\
\sum_{b \in B} \sum_{j \in Z} y_{j}^{b} & \geq \xi_{y_{0}}, \\
\sum_{b \in B} y_{j}^{b} & \leq \mu_{j}, \forall j \in Z, \\
y_{j}^{b} & \in\{0,1\}, b \in B, j \in Z .
\end{aligned}
$$

Constraint 4.2 requires $\mathcal{T}_{\max }$ to be greater than or equal to the maximal travel cost incurred by all buses and is to be minimized on Constraint (4.1). Constraints (4.3) and (4.4) are the bus and shelter capacity constraints, respectively, which ensure that all evacuees are transported and shelter capacities are respected. Constraint 4.5 represents whether the bus $b$ travels from source to sink $j$ ( or travels back from the sink $j$ to the source).

For the solution status and the proof of its decision version, similar to Theorem 4.1 as mentioned earlier, we have,

Theorem 4.2. The decision version of the BEPP in a diminished evacuation network is $\mathcal{N P}$-complete.

For the upper bound of the evacuation duration on the BEPP as in [12], four different heuristics algorithms were presented, three with the precomputed tourlists and the fourth without any precomputed tourlists. In the fourth algorithm, the assignment of transitvehicles begins with the best possibility to bring one evacuee back from the sink to the source and is continued iteratively, respecting the sink capacity constraints. It dominates the rest in evacuation duration and is equivalent to the nearest sink approach as in [4]. So, we prefer it as the dominating vehicle assignment for the network as in Example 4.4

Observation 4.3. Let $\xi_{y_{0}}$ and $\mu\left(z_{i}\right)$ be the number of evacuees at the super pickup node $Y_{0}$ and the sink capacities, respectively. Consider $\tau_{k}=\min \left\{\tau_{01}, \tau_{02}, \ldots, \tau_{0 n}\right\}$ as the nearest sink $z_{k}$ in the network. Let the extended sink capacity to the nearest sink be $\mu\left(z_{k}\right) \geq \sum_{j=1}^{n} \mu\left(z_{i}\right)$. Then such evacuation network $s-Z$ be reduced to an $s-z$ network with the minimum possible evacuation duration. For this, the estimated evacuation duration becomes $\left(2 \xi_{y_{0}}-1\right) \tau_{k}$. 


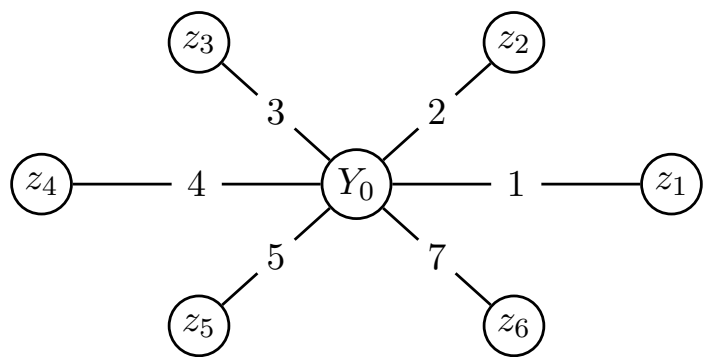

FiguRE 2. An instance of a bus-based evacuation problem in a diminished network.

Example 4.4. Consider a diminimshed evacuation network as in Figure 2, Let $\xi_{y_{0}}=26$ with $\mu\left(z_{i}\right)=\{7,6,5,4,3,2\}$ for $i=\{1,2, \ldots, 6\}$. Their respective transit times $\tau_{0 j}$ from $Y_{0}$ are shown along with the figure.

Then the tour plan for $|B|=1$ is given by $14 \tau_{01}+12 \tau_{02}+10 \tau_{03}+8 \tau_{04}+6 \tau_{05}+\tau_{06}$ with $\mathcal{T}_{\max }=137$. But if $|B| \geq 26$, then $\mathcal{T}_{\max }=7$. For $1<|B|<26$, consider $|B|=13$, as an arbitrary. Then the tour plan becomes $2 \tau_{01}+2 \tau_{02}, 2 \tau_{01}+2 \tau_{04}, 2 \tau_{02}+2 \tau_{04}, 2 \tau_{02}+2 \tau_{05}$ and $2 \tau_{02}+\tau_{07}$ for the buses $\left\{B_{1}, B_{2}, B_{3}, B_{4}, B_{5}\right\},\left\{B_{6}, B_{7}\right\},\left\{B_{8}, B_{9}\right\},\left\{B_{10}, B_{11}, B_{12}\right\}$ and $\left\{B_{13}\right\}$, respectively. For this, the effective $\mathcal{T}_{\max }=14$.

If this $s-Z$ network is replaced by an $s-z$ network having sufficient sink capacity at $z_{1}$ as requested by the given demand, then the tour plan for $|B|=1$ be reduced to $51 \tau_{01}$ with $\mathcal{T}_{\max }=51$. But for $|B|=13$, the respective $\mathcal{T}_{\max }=3$. However, for $|B| \geq 26$, it becomes 1 . This is why a single sink having sufficient capacity is more appropriate for the transit-based evacuation planning problem.

\section{Conclusions}

Proper planning of transit-vehicles within an integrated evacuation network might be helpful to reduce the massive loss of the people and the socio-economic damage during different disasters. It is beneficial for their normalcy. Planning a bus-based evacuation is an extremely rich problem. In general, a challenging task.

We have presented a compact overview concerning different solution strategies and the optimization approaches for the transit-based evacuation planning problem in an integrated network topology. Flow maximization and/or time minimization on the transshipments are highly affected by the arrival and assignment pattern of the evacuees. However, depending upon the optimization objectives and the nature of the network topology, it needs several extensions and should be investigated further.

Regardless of many directions of evacuation strategies, we have restricted mainly to the computationally acceptable research domain on the network flow optimization for the transit-based evacuation system in an integrated network topology. Most of the problems in this category are $\mathcal{N} \mathcal{P}$-hard in nature and demand the various computational techniques with acceptable approximations. Such $\mathcal{N} \mathcal{P}$-hard transit-dependent models and the solution strategies demanded by them have rarely been considered in the literature however most of the evacuation regions rely on transit-vehicles. In a real evacuation scenario, an optimizer has to address the characteristics of the diversified and heterogeneous vehicles including different commodities that are rather complicated from their computational aspects. 
Conflict of Interest: The authors declare no conflict of interest.

Acknowledgments: The first author wants to acknowledge the University Grants Commission, Nepal, for the Ph.D. research fellowship. The authors would also like to thank the anonymous referees for their valuable suggestions to improve the quality of this paper.

\section{REFERENCES}

[1] I. M. Adhikari and T. N. Dhamala, Minimum clearance time on the prioritized integrated evacuation network, American Journal of Applied Mathematics, Vol. 8(4), pp 207-215, 2020.

[2] I. M. Adhikari and T. N. Dhamala, An insight on the evacuation planning optimization problems on transit-based system, In: 11th Triennial Conference of Association of Asia Pacific Operational Research Society, APORS-2018, pp 132-135, 2018.

[3] I. M. Adhikari and T. N. Dhamala, Dominance vehicle routine in transit dependent evacuation scenario, In: National Conference on Mathematics and its Applications (NCMA-2017), pp 54-60, 2017.

[4] I. M. Adhikari, U. Pyakurel, and T. N. Dhamala, An integrated solution approach for the time minimization evacuation planning problem, International Journal of Operation Research, Vol. 17(1), pp 27-39, 2020.

[5] D. R. Bish, Planning for a bus-based evacuation, OR Spectrum, Vol. 33, pp 629-654, 2011.

[6] T. N. Dhamala and I. M. Adhikari, On evacuation planning optimization problems from transit-based perspective, International Journal of Operation Research, Vol. 15(1), pp 29-47, 2018.

[7] T. N. Dhamala, I. M. Adhikari, H. N. Nath, and U. Pyakurel, Meaningfulness of OR models and solution strategies for emergency planning, In: Living Under the Threat of Earthquakes, Edited by Kruhl, J.H., Adhikari, R. and Dorka, U.E., Springer Natural Hazards, pp 175-194, 2018.

[8] T. N. Dhamala, U. Pyakurel, and S. Dempe, A critical survey on the network optimization algorithms for evacuation planning problems, International Journal of Operation Research, Vol. 15(3), pp 101-133, 2018.

[9] R. C. Dhungana and T. N. Dhamala, Flow improvement in evacuation planning with budget-constrained switching costs, International Journal of Mathematics and Mathematical Sciences, Vol. 3, pp 1-10, 2020.

[10] L.R. Ford and D. R. Fulkerson, Flows in Networks. Princeton University Press, Princeton, NJ, 1962.

[11] M. Goerigk and B. Grün, A robust bus evacuation model with delayed scenario information, OR Spectrum, Vol. 36, pp 923-948, 2014.

[12] M. Goerigk, B. Grün, and P. Heßler, Branch and bound algorithms for the bus evacuation problem, Computers and Operations Research, Vol. 40, pp 3010-3020, 2013.

[13] B. Hoppe and E. Tardos, The quickest transshipment problem, Mathematics of Operation Research, Vol. 25(1), pp 36-62, 2000.

[14] J. Hua, G. Ren, Y. Cheng, and B. Ran, An integrated contraflow strategy for multimodal evacuation, Hindawi Publishing Corporation, Mathematical Problems in Engineering, Vol. 2014, 2014.

[15] B. Jamei, Transportation actions to reduce highway evacuation times under natural disasters, Ph.D. Thesis, Virginia Polytechnic Institute and State University, 1984.

[16] E. I. Kaisar, L. Hess, and A. B. P. Palomo, An emergency evacuation planning model for special needs populations using public transit systems, Journal of Public Transportation, Vol. 15(2), pp 45-69, 2012.

[17] M. Li, J. Xu, L. Wei, X. Jia, and C. Sun, Modeling a risk-based dynamic bus schedule problem under no-notice evacuation incorporated with dynamics of disaster, supply, and demand conditions, Hindawi Publishing Corporation, Journal of Advanced Transportation, Vol. 2019, 2019.

[18] T. Litman, Lessons from Katrina and Rita: What Major Disasters can Teach Transportation Planners, Journal of Transportation Engineering, Vol. 132(1), pp 11-18, 2006.

[19] N. Megiddo, Optimal flows in networks with multiple sources and sinks. Mathematical Programming, Vol. 7, pp 97-107, 1974.

[20] N. Megiddo, A good algorithm for lexicographically optimal flows in multi-terminal networks. Bulletin of the American Mathematical Society, Vol. 83, pp 407-409, 1977. 
[21] E. Minieka, Maximal, lexicographic, and dynamic network flows. Operation Research, Vol. 21, pp 517$527,1973$.

[22] V. C. Pereira and D. R. Bish, Scheduling and routing for a bus-based evacuation with a constant evacuee arrival rate, Transportation Science, Vol. 49(4), pp 853-867, 2014.

[23] U. Pyakurel, M. Goerigk, T. N. Dhamala, and H. W. Hamacher, Transit dependent evacuation planning for Kathmandu valley: a case study, International Journal of Operations Research Nepal, Vol.5, pp 49$73,2015$.

[24] U. Pyakurel, H. N. Nath, S. Dempe, and T. N. Dhamala, Efficient dynamic flow algorithms for evacuation planning problems with partial lane reversal, Mathematics, Vol. 7(10), 993, 2019.

[25] U. Pyakurel, H. N. Nath, and T. N. Dhamala, Partial contraflow with partial reversals for evacuation planning, Annals of Operations Research, Vol. 283, pp 591-612, 2019.

[26] U. Pyakurel and T. N. Dhamala, Models and algorithms on contraflow evacuation planning network problems, International Journal of Operations Research, Vol. 12(2), pp 36-46, 2015.

[27] U. Pyakurel and T. N. Dhamala, Evacuation planning by earliest arrival contraflow, Journal of Industrial and Management Research, Vol. 13, pp 489-503, 2017.

[28] S. Rebnnack, S. Arulselvan, E. Lily, and P. M. Pardalos, Complexity analysis for maximum flow problem with arc reversals, Journal of Combinatorial Optimization, Vol. 19, pp 200-216, 2010.

[29] M. Schmidt and M. Skutella, Earliest arrival flows in network with multiple sinks, Discrete Applied Mathematics, Vol. 164, pp 320-327, 2014 